\title{
Chirped-pulsed Kerr solitons in the Lugiato-Lefever equation with spectral filtering
}

\author{
Xue Dong $\odot,{ }^{*}$ Christopher Spiess $\odot$, Victor G. Bucklew, and William H. Renninger \\ Institute of Optics, University of Rochester, Rochester, New York 14627, USA
}

(Received 3 June 2021; accepted 27 August 2021; published 15 September 2021)

\begin{abstract}
Optical Kerr resonators support a variety of stable nonlinear phenomena in a simple and compact design. The generation of ultrashort pulses and frequency combs has been shown to benefit several applications, including spectroscopy and telecommunications. The most common anomalous dispersion Kerr resonators can be accurately described by a well-studied mean-field Lugiato-Lefever equation (LLE). Recently observed highly chirped pulses in normal dispersion resonators with a spectral filter, however, cannot. Here, we examine the LLE in the normal dispersion regime modified with a Gaussian spectral filter (LLE-F). In addition to solutions associated with the LLE, we find stable highly chirped pulses. Solutions are strongly dependent on the filter bandwidth. Because of the large changes per round trip, the validity of the LLE-F fails over a large range of experimentally relevant parameters. While the mean-field approach leads to accurate predictions with respect to the nonlinearity coefficient and the dispersion, the dependence of drive power on loss deviates significantly from an experimentally accurate model, which leads to opportunities for Kerr resonators including frequency comb generation from low-Q-factor cavities.
\end{abstract}

DOI: 10.1103/PhysRevResearch.3.033252

\section{INTRODUCTION}

The generation of optical frequency combs in nonlinear Kerr resonators is attractive for a wide range of applications including spectroscopy, frequency synthesis, imaging, distance measurements, and telecommunications [1-8]. Solitons play a key role in the generation of coherent frequency combs in Kerr resonators. To date, such Kerr cavity solitons have been demonstrated in fiber [9], chip-scale [10], and bulk resonator [11] platforms. In contrast to traditional mode-locked lasers, Kerr resonators can support frequency combs over a wider range of frequencies with megahertz to terahertz line spacing $[12,13]$. Pulsed Kerr resonators are largely based on anomalous dispersion soliton formation, which is related to the anomalous dispersion solitons underlying common modelocked lasers. Sources based on anomalous dispersion solitons can be limited in key pulse parameters, including the duration and energy $[9,10,14,15]$. To address these limits, mode-locked laser researchers developed cavities with alternative dispersion profiles supporting different types of solitons, and recent efforts in fiber cavities and microresonators suggest that generating related soliton types can be valuable in Kerr resonators in a similar way. For example, stretched-pulse solitons in dispersion-managed cavities exhibit significantly improved pulse durations $[16,17]$.

\footnotetext{
*xdong18@ur.rochester.edu

Published by the American Physical Society under the terms of the Creative Commons Attribution 4.0 International license. Further distribution of this work must maintain attribution to the author(s) and the published article's title, journal citation, and DOI.
}

The expansion of frequency comb generation to normal dispersion resonators has enabled a wider variety of pulse shaping mechanisms and a wider range of cavity configurations. Researchers have demonstrated modulation instability, dark solitons, and switching waves [18-22] as well as brightpulse solutions enabled by interactions between different transverse mode families $[23,24]$ and higher-order dispersive and Raman interactions [25-30]. In mode-locked lasers, it is well known that a strong spectral filter is required to generate bright chirped pulses which can support ultrashort pulses with large energies [31-33]. In Kerr resonators, this concept was initially explored in normal dispersion microresonators with an intrinsically wavelength-dependent quality factor [34]. More recently, in normal dispersion fiber resonators with strong spectral filtering, highly chirped pulses were observed, clearly demonstrating the importance of spectral filtering as well as the potential for energy enhancement [35].

Traditional anomalous dispersion Kerr resonators are accurately modeled using a nonlinear Schrödinger-type equation for the waveguide, in addition to periodic boundary conditions accounting for the loss and drive [36-38]. This Ikeda-type model can be approximated by an averaged-cavity LugiatoLefever equation (LLE), in the limit of negligible changes in the field over one roundtrip in the cavity [39-41]. Solutions of the LLE agree well with experiments [42-44] and have been used for the study of stability, threshold powers, and parameter scaling laws $[43,45-47]$. In addition, spectral filtering added to the LLE with anomalous dispersion has been found to play a role in soliton oscillations and interactions [48,49]. Averaged-cavity models have also been applied toward normal dispersion cavities, accounting for third order dispersion (TOD) and Raman as appropriate [25-30]. While modulation instability has been observed with notch filtering in Ikeda[50] and LLE-based [51] models and bright pulses with time 
oscillating structure have been observed with spectral filtering in an LLE-based model [34], highly chirped pulses have not been observed. Experimentally observed highly chirped pulses are accurately predicted by an Ikeda-type model with Gaussian spectral filtering (Ikeda-F) applied with the boundary condition [35]. However, because of the large changes per round trip of the pulses, it is not clear that this system can be approximated by an averaged-cavity model and that the resulting scaling laws or conventional wisdom would still apply. In a quadradic nonlinear two-dimensional planar cavity, for example, the experimentally accurate model and the approximate model were found to deviate at larger powers [52]. More generally, in contrast to the general Ginzburg-Landau master equation models for mode-locked lasers with a filter in normal dispersion [53], the general LLE model for Kerr resonators in normal dispersion with a spectral filter (the LLE-F) has rarely been investigated.

Here, we numerically examine the LLE-F in the normal dispersion regime. In addition to known solutions found without a filter, we observe stable highly chirped pulses across a wide range of parameters with the appropriate choice of spectral filter. The drive power and other system parameters in which stable chirped pulses are found are strongly dependent on the filter bandwidth. We find that the averaged-cavity approach fails quantitatively for chirped pulses owing to the large changes per round trip inherent in the pulse dynamics. Scaling laws derived from the LLE-F are also evaluated in comparison with the experimentally accurate Ikeda-F model. We show that useful scaling dependencies on nonlinearity and dispersion remain accurate despite large changes per round trip. In contrast, we find that the dependence of drive power on loss is much weaker than the cubic dependence predicted by the averaged cavity model. This deviation leads to exciting implications for Kerr resonator designs based on chirped-pulse operation including the potential for useful comb generation in low-Q-factor resonators.

\section{MODEL AND METHOD}

Chirped-pulse solitons are found in driven normal dispersion Kerr resonators with a Gaussian spectral filter. This experiment can be accurately modeled with an Ikeda-type system of equations including an equation describing the propagation of the slowly varying envelope of the field inside the resonator waveguide and a periodic boundary condition accounting for the discrete application of the drive, losses, and the spectral filter [35]. The relative detuning of the laser from the peak of the cavity resonance leads to a relative phase between the drive and the propagating electric field. This detuning can be modeled equivalently by either the waveguide propagation equation or the boundary condition (see Appendix A). Pulse propagation in the resonator waveguide, including the relative phase detuning, is modeled by a detuned nonlinear Schrödinger equation:

$$
\frac{\partial A}{\partial z}=-i \frac{\overline{\beta_{2}}}{2} \frac{\partial^{2} A}{\partial t^{2}}+i \gamma|A|^{2} A-i \delta A,
$$

where $A$ is the slowly varying electric field envelope, $z$ is the propagation coordinate, $t$ is time in the moving frame, $\overline{\beta_{2}}$ is the average group-velocity dispersion, $\gamma$ is the Kerr nonlinear coefficient, and $\delta$ is the cavity detuning per unit length.

The periodic boundary condition incorporating the drive, spectral filter, and loss is described as

$$
\begin{aligned}
A_{n+1}(\omega)= & A_{n}(\omega) \exp \left\{-\left[\frac{\left(\omega-\omega_{0}\right)^{2}}{f_{\mathrm{tot}}^{2}}+\frac{\alpha_{\mathrm{tot}}}{2}\right]\right\} \\
& +\sqrt{D_{\mathrm{tot}}} \delta\left(\omega-\omega_{0}\right),
\end{aligned}
$$

where $A_{n}(\omega)$ represents the electric field after propagation through the waveguide of the $n$th roundtrip, $A_{n+1}(\omega)$ represents the electric field before propagation through the waveguide of the $(n+1)$ th roundtrip, $\alpha_{\text {tot }}$ is the total loss in the cavity (note that fiber loss is negligible in comparison with component losses in fiber Kerr resonators), $D_{\text {tot }}$ is the total input coupled drive power, $\omega_{0}$ is the drive frequency, and $f_{\text {tot }}$ represents the spectral filter bandwidth applied each roundtrip.

The Ikeda-type system of equations can be approximated by a single continuous partial differential equation if the electric field envelope exhibits negligible evolution in the cavity [34,39-41].The resultant equation derived following the approach used to develop the LLE model for Kerr resonators, with the addition of a spectral filter, is expressed as

$$
\frac{\partial A}{\partial z}=-\frac{\alpha}{2} A+\left(-i \frac{\overline{\beta_{2}}}{2}+\frac{1}{f^{2}}\right) \frac{\partial^{2} A}{\partial t^{2}}+i \gamma|A|^{2} A-i \delta A+\sqrt{D},
$$

where $\alpha$ is the loss per unit length, $f$ represents the filter bandwidth per unit length, and $D$ is the input coupled drive power per unit length. The LLE-F is the simplest continuous field model incorporating all the physical effects of chirped-pulse supporting Kerr resonators.

While the Kerr-resonator-specific Ikeda-type model is known to accurately model experiments [35], the approximate single-field LLE-F model is simple and potentially relevant to other physical systems modeled by the nonlinear Schrödinger equation. In contrast to the general Ginzburg-Landau master equation models for mode-locked lasers with a filter [53], this LLE-F master equation for Kerr resonators with a spectral filter remains relatively unexplored. In what follows, the LLE$\mathrm{F}$ is examined and compared with the Ikeda-F model, with emphasis on practically important chirped-pulse solitons.

\section{RESULTS}

\section{A. Chirped-pulse solitons in the LLE-F}

In this section, we numerically examine stable solutions of the LLE and LLE-F. The dispersion, nonlinearity, spectral filter, and loss per unit length are chosen to be consistent with relevant experimental conditions [35] (see Appendix A). After fixing these parameters, the simulations are examined as a function of the remaining, experimentally tunable parameters: the drive power and frequency detuning (Fig. 1). Stable solutions are found with and without a filter. The trivial continuous wave $(\mathrm{CW})$ solutions are indicated with white, and stable nontrivial solutions are indicated by color in Fig. 1.

The nontrivial solutions found in the LLE are classified as dark solitons (gray region in Fig. 1), consistent with previous studies $[45,54,55]$. Around the threshold power, the spectrum 


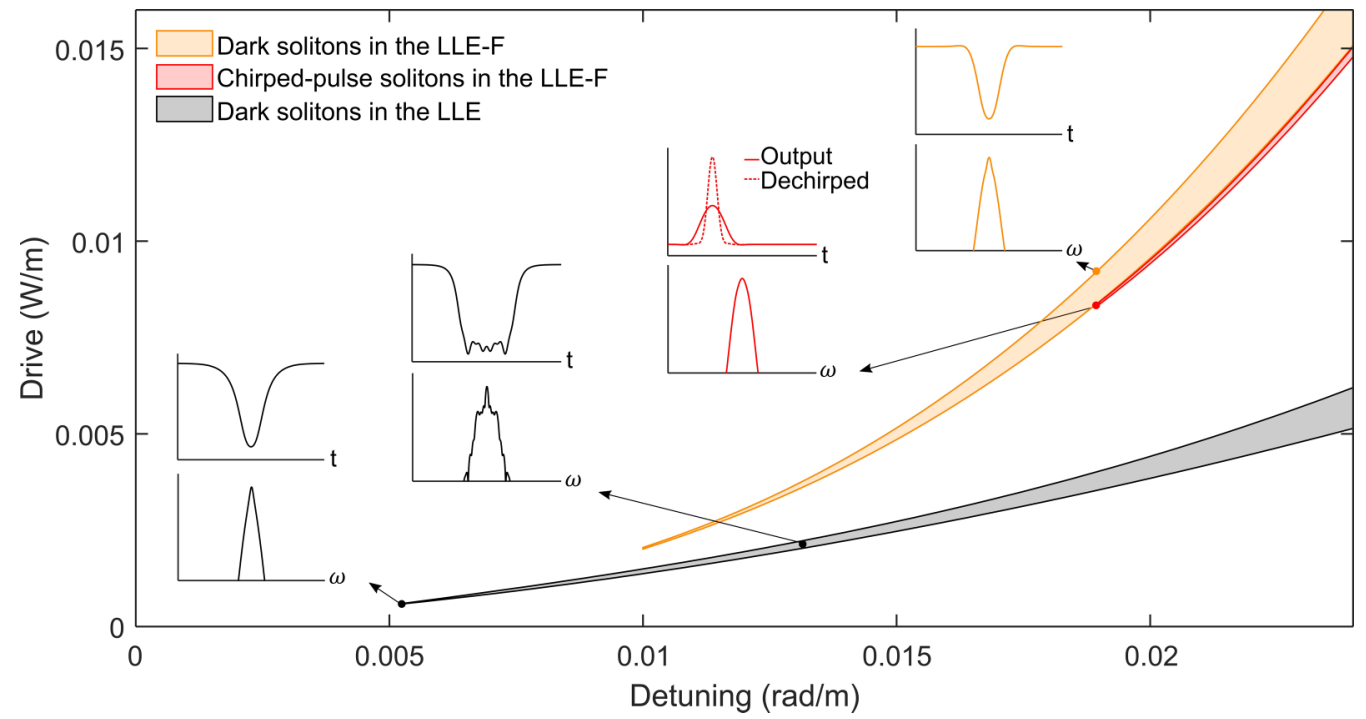

FIG. 1. Location of stable nontrivial solutions in drive-detuning parameter space of normal dispersion cavity with (LLE-F) and without (LLE) spectral filtering. The gray region represents dark solitons of the LLE, the orange region represents dark solitons of the LLE-F with a $14.5 \mathrm{~nm}$ per unit length spectral filter, and the red region represents chirped-pulse solitons in the LLE-F with the same filter. Pulse and spectral profiles of four representative stable solutions are inset.

of the dark soliton is smooth (see the inset temporal and spectral profiles). As the drive frequency detuning increases, the dark soliton spectrum develops structure. In previous reports, solutions featuring such a structure have been referred to as complex dark solitons [45].

With the addition of a spectral filter, in the LLE-F, stable dark solitons are also observed (orange in Fig. 1). For the same detuning parameter as in the LLE, the dark solitons in the LLE-F are stable for a higher drive power. The relative deviation of the drive power increases with larger values of detuning. In addition, the dark solitons observed in the LLE-F are less complex than those in the LLE, with smoother spectra. In the LLE-F, in addition to dark solitons, bright solitons are also found to be stable on the low drive boundary of the stable dark soliton solutions (red in Fig. 1). In contrast, for the LLE, only the trivial $\mathrm{CW}$ solutions are observed at drive powers lower than the dark soliton region. The bright pulses have a unique threshold drive power that is larger than the threshold power for dark solitons.

The stable bright solitons feature a quadratic spectral phase that can be removed through the application of anomalous group-velocity dispersion. In other words, the bright pulses are chirped and can be dechirped by removing the excess linear phase to have a shorter pulse duration and a higher peak power (e.g., see Fig. 1 red inset). Like the dark solitons in the LLE-F, chirped pulses exhibit a smooth temporal and spectral profile, without the prominent time oscillating structure observed with bright-pulse solutions of the LLE with alternative modifications (e.g., with higher-order dispersion or Raman scattering) [25-30]. In analogy with those in mode-locked lasers [53], chirped-pulse solitons in Kerr resonators can be understood as two interlocking front solutions (also known as domain walls or switching waves), stabilized by spectral filtering (see Appendix B for further analysis). As detailed further in Sec. III C, the key performance parameters for chirpedpulse solitons, such as the spectral bandwidth and the drive threshold power are strongly dependent on the bandwidth of the spectral filter.

\section{B. LLE-F vs Ikeda-F}

Chirped-pulse Kerr resonator solitons were numerically observed in the experimentally accurate Ikeda-type model for normal dispersion Kerr resonators with a filter (Ikeda-F) [35]. Here, we compare chirped-pulse soliton solutions of the Ikeda-F with those of the LLE-F. For the LLE-F, all the physical effects are evenly distributed in the propagation direction, whereas for the Ikeda-F, the nonlinear and dispersive fiber is separated from the drive, loss, and spectral filter, as illustrated in Fig. 2. The chirped-pulse solitons of the LLE-F and Ikeda-F are examined for the same effective dispersion, nonlinearity, filter bandwidth, and drive power per unit length. The detuning for each model is chosen such that chirped pulses are stable in each model, resulting in a larger detuning in the Ikeda-F model in comparison with that in the LLE-F.

While in the continuous LLE-F model, the root-meansquare (RMS) bandwidth of the spectrum remains constant [Fig. 2(a)], the chirped-pulse soliton exhibits rich intracavity dynamics in the Ikeda-F system [Fig. 2(b)]. In the Ikeda-F system, the bandwidth increases monotonically in the nonlinear waveguide and is decreased to its original bandwidth each round trip by the discrete spectral filter. When the spectrum is broadest, it features side peak structure which is absent in the smooth solution of the LLE-F [Figs. 2(c) and 2(d)]. The pulse duration of the chirped-pulse soliton in the Ikeda-F (LLE-F) system can be reduced by a factor of 3.8 (2.4) by dechirping [Figs. 2(f) and 2(e)]. Temporal oscillations are apparent on the wings of the chirped pulses in the Ikeda-F system but are not present in the LLE-F. The temporal oscillations are removed after the dechirping process, indicating that they are not a consequence of the spectrum. The soliton pulse energy and dechirped-pulse duration are comparable with a pulse energy 
LLE-F

(a) $+\beta 2, \gamma$, loss, drive, and filter
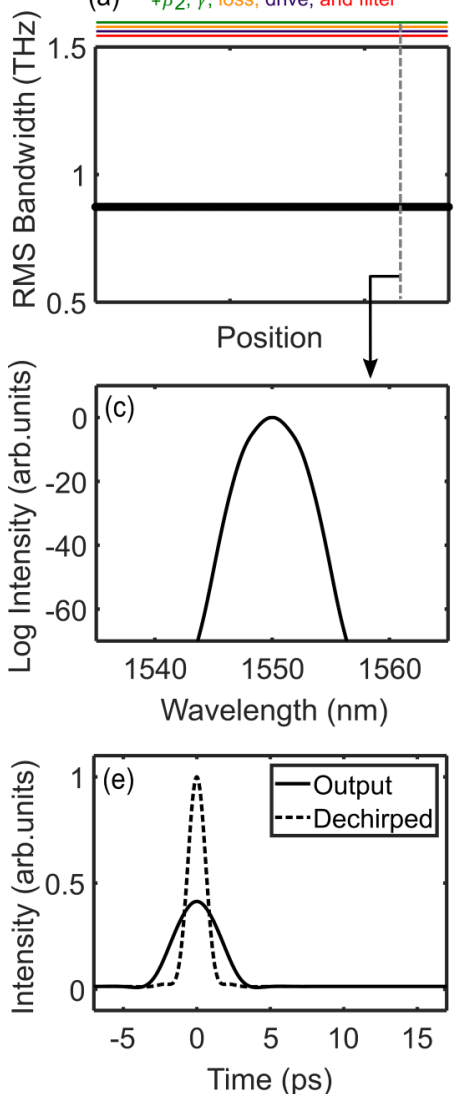

Ikeda-F
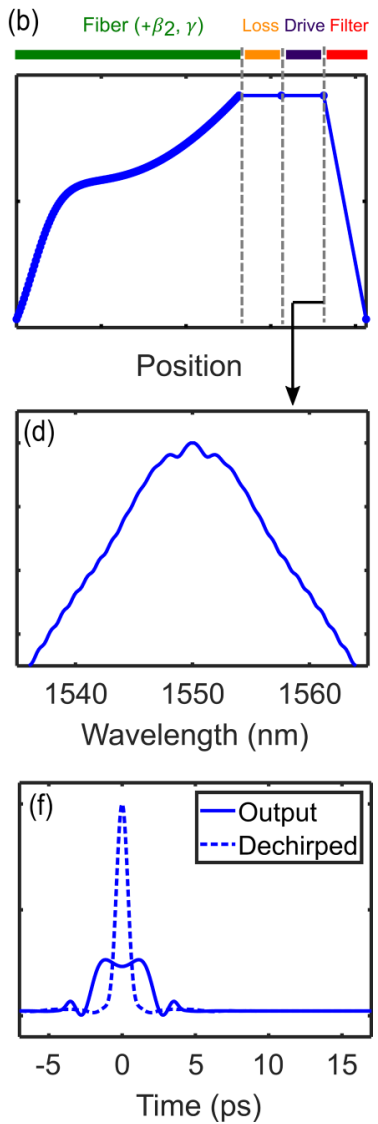

FIG. 2. Comparison of chirped-pulse solutions in the LLE-F and Ikeda-F models. (a) Intracavity evolution of the spectral bandwidth, (c) the output spectrum, and (e) the output chirped (solid) and dechirped (dashed) pulse for chirped solitons of the LLE-F with drive $=0.25 \mathrm{~W} / \mathrm{m}$, detuning $=0.07 \mathrm{rad} / \mathrm{m}$, and with a $14.5-\mathrm{nm}$ per unit length Gaussian spectral filter and (b) intracavity evolution of the spectral bandwidth, (d) the output spectrum, and (f) the output chirped (solid) and dechirped (dashed) pulse for chirped solitons of the Ikeda-F with drive $=0.25 \mathrm{~W} / \mathrm{m}$, detuning $=0.1 \mathrm{rad} / \mathrm{m}$, and with a 14.5- nm per unit length Gaussian spectral filter .

(pulse duration) of $221 \mathrm{pJ}(0.9 \mathrm{ps})$ for the Ikeda-F model and $126 \mathrm{pJ}$ (1.4 ps) for the LLE-F model.

The chirped-pulse solutions of the Ikeda-F model have inherent practical benefits stemming from the intracavity evolution of the pulse parameters. If the pulse parameters evolve in the cavity, the output coupling location can be chosen such that the pulse parameters are desirable. For example, in this case, the soliton has its broadest bandwidth after the fiber waveguide section [Fig. 2(d)], which is broader than the averaged-cavity equivalent spectrum [Fig. 2(c)]. This explains why the dechirped-pulse duration is short for the Ikeda-F model case. The chirped solitons of the Ikeda-F model have been shown to support high energy [35], which also suggests that the breathing evolution may be beneficial to energy storage.

The Ikeda-F model, given parameters which accurately model experimental results (as in Ref. [35]), supports chirped solitons featuring significant intracavity evolution (as in

Fig. 2). However, the LLE-F is valid in the limit of negligible intracavity evolution. To determine the degree and range of validity of the LLE-F model, we examine the regions of parameter space in which chirped-pulse solutions exist in both the LLE-F and the Ikeda-F models (Fig. 3). As in Fig. 1, the dispersion, nonlinearity, and loss are chosen based on experimental parameters and fixed. The locations of stable solutions are plotted for each model in Fig. 3 as a function of drive power and frequency detuning, for two different filter bandwidths. To focus and simplify our analysis, only chirpedpulse solutions are shown in color in Fig. 3, with all other solutions indicated with white. The system parameters where stable chirped pulses exist are close for the LLE-F and Ikeda-F models for lower drive and detuning values, near the threshold drive power. In contrast, for larger drive and detuning values, the system parameters for stable chirped pulses deviates, and the LLE-F model predicts chirped pulses at higher drive powers for the same detuning as the Ikeda-F model. These results suggest that the LLE-F approximation works well for small drive and detuning values (near threshold) but begins to break down at higher powers.

To gain some insight into the variation of validity of the LLE-F vs detuning, we examine the pulse evolution as a function of detuning (see Appendix C). For larger detuning values, the total change of the electric field is larger, demonstrating a clear correlation between the strength of the pulse evolution and the validity of the master equation (averaged-cavity) LLEF model (Fig. 4). Because the LLE-F becomes strictly invalid for a wide range of parameter space, it is important to examine in more detail what implications this has for basic scaling law predictions and conventional wisdom for chirped-pulse cavity solitons, which can be found in Sec. III D. Notice that, including the range of validity of the LLE-F, all the pulse and system parameters have a clear dependence on the spectral filter bandwidth.

\section{Dependence on the filter bandwidth}

The spectral filter is important for the generation of chirped-pulse solitons. For example, as illustrated by Fig. 1, chirped pulses are not observed without spectral filtering. Moreover, the specific bandwidth of the filter determines key pulse and system parameters. To evaluate the dependence on the spectral filter bandwidth in the LLE-F and Ikeda-F models, numerical simulations are performed with the identical system parameters for two distinct filter bandwidths (Fig. 3). For the same drive power, the spectral bandwidths of chirpedpulse solitons in both models are broader with a broader spectral filter (see Fig. 3 insets for example). In addition, the corresponding dechirped pulses have shorter pulse durations. Finally, the corresponding pulse energy is higher with a broader filter in both models. With a broader filter bandwidth, the drive threshold power is significantly higher for generating chirped-pulse solitons in both models. Greater than 25 times more drive power is required for generating stable chirped pulses in the LLE-F for a filter bandwidth that is 1.4 times broader. For the Ikeda-F model, $\sim 10$ times higher threshold power is required. If the filter bandwidth is too broad, chirped pulses are not stable in the Ikeda-F model [35]. In addition, for broader filter bandwidths (including the $20.3 \mathrm{~nm}$ result 


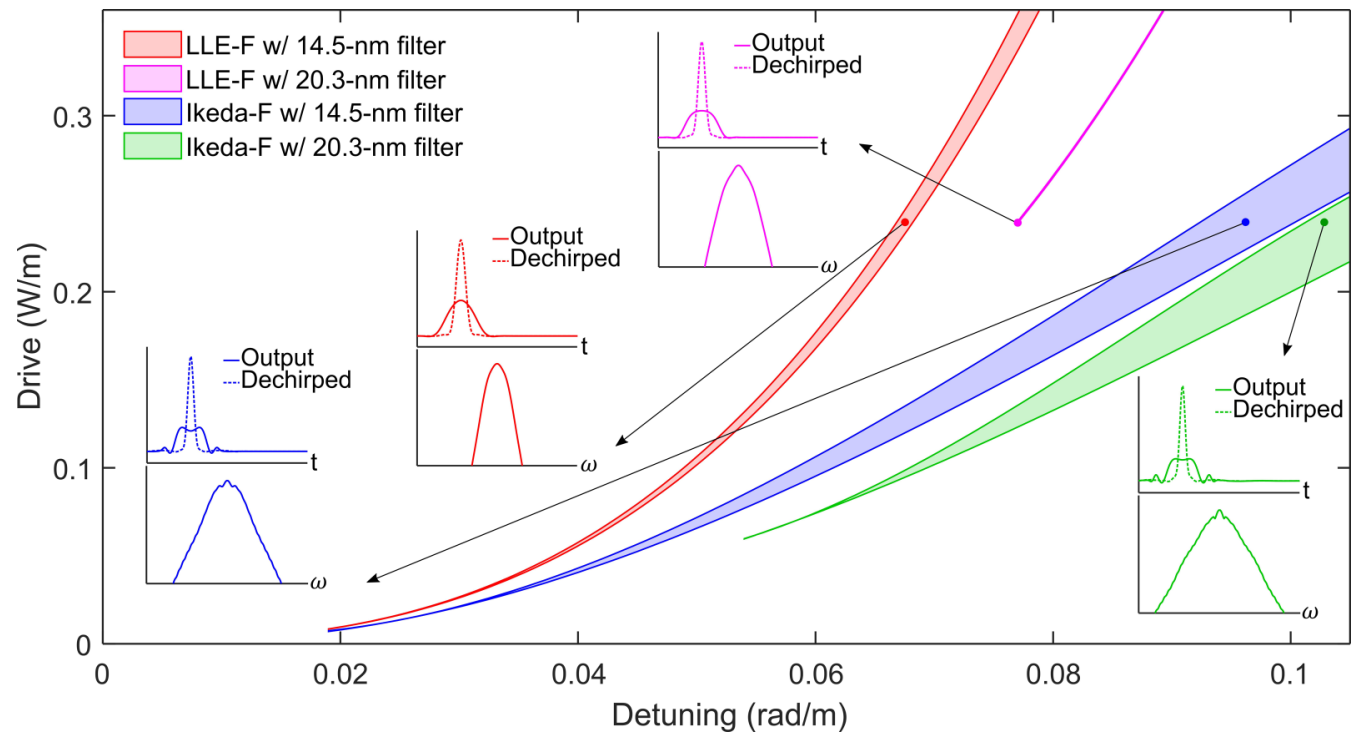

FIG. 3. Location of stable chirped pulses in drive-detuning parameter space for the LLE-F and Ikeda-F models. Stable chirped pulses are found in the LLE-F with $14.5 \mathrm{~nm}(20.3 \mathrm{~nm})$ per unit length filter in the red (magenta) regions. Stable chirped pulses are found in the Ikeda-F with $14.5 \mathrm{~nm}(20.3 \mathrm{~nm})$ per unit length filter in the blue (green) regions. Pulse and spectral profiles of four representative chirped pulses at a fixed drive power are inset.

shown), the minimum change of the field per roundtrip, found at threshold, is large (e.g., $\sim 1.6$ for $20.3 \mathrm{~nm}$ ). Consequently, for a range of filter bandwidths, the LLE-F is strictly invalid for any drive and detuning values for which the stable chirped pulses exist. Therefore, it is important to investigate the theoretical implications of this deviation.

\section{System parameter scaling dependence}

A continuous model yields simple analytical relationships between the required system and pulse parameters which are valuable for the design and understanding of chirped-pulse solitons. Here, we examine the validity of these simple scaling laws in the limit in which the LLE-F becomes strictly invalid, for parameters where chirped-pulse solitons are stable with

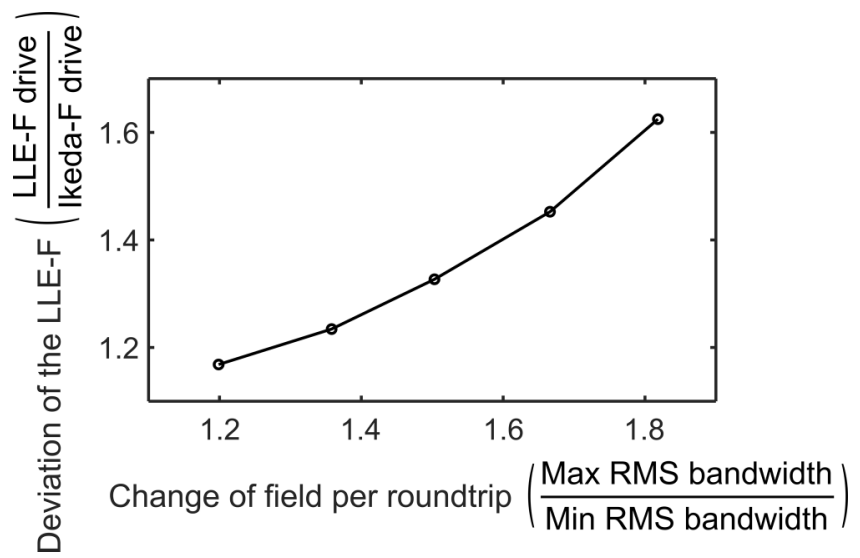

FIG. 4. The deviation of the required drive power of stable chirped pulses in the LLE-F and Ikeda-F models vs the total change of the field during one roundtrip in the cavity. Change per round trip is calculated as the ratio between the highest and the lowest intracavity root-mean-square (RMS) bandwidth. large intracavity evolution. Following the approach outlined in Ref. [35], scaling laws for the system parameters can be developed from the LLE-F model and are

$$
D_{n 0}=D_{\text {tot }} \frac{\gamma L}{\alpha_{\text {tot }}^{3}}, \quad f_{n 0}=f_{\text {tot }} \sqrt{L\left|\overline{\beta_{2}}\right|}, \quad \text { and } \quad \delta_{n 0}=\frac{\delta_{\text {tot }}}{\alpha_{\text {tot }}},
$$

where $\delta_{\text {tot }}, D_{n 0}, f_{n 0}$, and $\delta_{n 0}$ are the total detuning, normalized drive, normalized spectral filter bandwidth, and normalized total detuning, respectively. If the three normalized parameters are known for any stable chirped-pulse soliton solution (e.g., using Fig. 3), then another stable chirped-pulse soliton can be obtained if the parameters (e.g., drive, nonlinearity, loss) are scaled according to Eq. (4). The pulse parameters also scale with the system parameters as

$$
P_{n 0}=P \frac{\gamma L}{\alpha_{\mathrm{tot}}} \quad \text { and } \quad \Delta T_{n 0}=\Delta T \sqrt{\frac{\alpha_{\text {tot }}}{L\left|\overline{\beta_{2}}\right|}},
$$

where $\Delta T, P, P_{n 0}$, and $\Delta T_{n 0}$ are the pulse duration, peak power, normalized peak power, and normalized pulse duration, respectively.

To test the validity of the simple LLE-F-derived scaling laws in the limit of large intracavity evolution, we numerically examine the relationships between the system and pulse parameters in the Ikeda-F model in comparison with the scaling laws. First, we examine the dependence of the system and pulse parameters on the nonlinear coefficient. From Eq. (4), among the system parameters, only the drive scaling law is predicted to depend on the nonlinear coefficient. To test this dependence, the other parameters are fixed, and the drive power is swept until stable chirped-pulse solitons are found. The drive power obtained, the corresponding peak power, and pulse duration are plotted in Fig. 5 along with the scaling laws [Eqs. (4) and (5)] obtained from the LLE-F. The drive [Fig. 5(a)] and peak power [Fig. 5(c)] scale inversely with the nonlinearity, and the pulse duration remains con- 

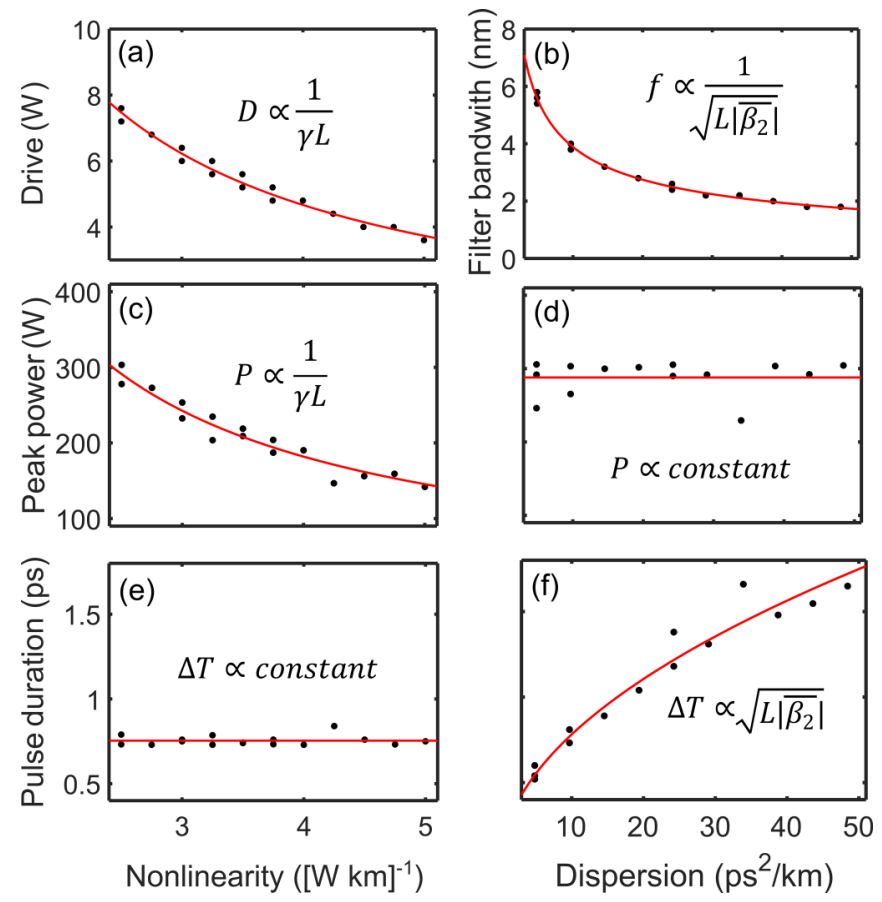

FIG. 5. Comparison of the chirped-pulse system and pulse parameters from the Ikeda-F model (points) with the scaling laws derived from the LLE-F (red lines). From Eq. (4), (a) the drive power varies with nonlinearity, and (b) the full- width at half maximum filter bandwidth varies with dispersion. From Eq. (5), (c) the peak power decreases, and (e) the pulse duration is constant with increasing nonlinearity, and (d) the peak power is constant, and (f) the pulse duration increases with increasing dispersion. For variations in nonlinearity and dispersion, the scaling laws agree well with the Ikeda-F model.

stant [Fig. 5(e)], as predicted by the LLE-F scaling laws. Remarkably, this global dependence on nonlinearity predicted by the LLE-F is accurate for the Ikeda-F system despite large changes of the field in the cavity of $\sim 2$.

The dependence of the system and pulse parameters on the dispersion is examined in Fig. 5. From Eq. (4), among the system parameters, only the filter scaling law is predicted to depend on the dispersion. To test this dependence, the other parameters are fixed, and the filter bandwidth is swept until stable chirped-pulse solitons are found. The filter bandwidth obtained, the corresponding peak power, and pulse duration are plotted in Fig. 5 along with the scaling laws [Eq. (4) and (5)] obtained from the LLE-F. The spectral filter bandwidth has an inverse square root dependence on the total dispersion of the cavity [Fig. 5(b)], the pulse duration has a square root dependence on the total dispersion of the cavity [Fig. 5(f)], and the peak power of the pulse remains constant with dispersion [Fig. 5(d)]. Again, remarkably, the dispersive trends also agree with the theoretical predictions from the LLE-F model despite large changes of the field in the cavity of $\sim 2$. This is beneficial practically because these relationships can be used to determine the appropriate choice of spectral filter bandwidth and drive power for generating chirped pulses.

Finally, we examine the system and pulse parameter dependence on the resonator loss. From Eq. (4), the drive and
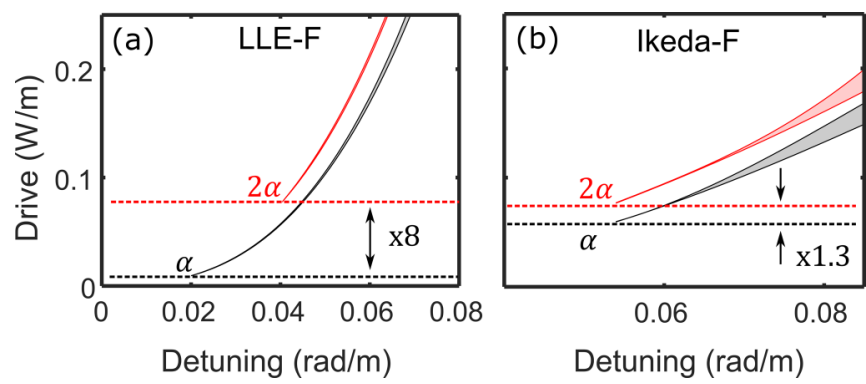

FIG. 6. Location of stable chirped pulses in drive-detuning parameter space for the (a) LLE-F and (b) Ikeda-F models for different losses per unit length $\alpha$. The change in the required drive is much lower (1.3) for the Ikeda-F model than that predicted by the LLE-F model [8 from Eq. (4)].

detuning scaling laws both have a dependence on loss. Therefore, to examine how the system varies with loss, we fix the nonlinearity, dispersion, and spectral filter and examine the location of stable chirped-pulse solitons in parameter space as a function of both drive and detuning for two different loss values for both the Ikeda-F and LLE-F models (Fig. 6). In the LLE-F system [Fig. 6(a)], when the loss is doubled, the drive power is eight times larger, and the detuning is two times larger, which agrees with the corresponding scaling laws [Eq. (4)]. However, in stark contrast, for the experimentally accurate Ikeda-F model, when the loss doubles, the drive increases by a small fraction, and the detuning remains nearly unchanged [Fig. 6(b)]. This large deviation from the scaling laws derived from the LLE-F stems from the large evolution of the field in the cavity in the Ikeda-F system. From a practical perspective, this deviation implies that the drive power does not have a strong dependence on loss for chirped-pulse solitons. This leads to the intriguing implication that chirped pulses can be generated in very low-Q cavities without significant drive power, in contrast to conventional wisdom regarding microresonator systems.

\section{DISCUSSION}

Here, we report on a chirped-pulse solution to a general master-equation model for Kerr resonators, the LLE-F. This is a strongly dissipative system in which stable pulse formation relies on a strong double balance of amplitude and phase modulations. The analogous undriven Ginzburg-Landau equation with gain is a similarly highly dissipative system which also supports chirped pulses. Unlike the present LLE-F in the normal dispersion region, the Ginzburg-Landau system in the normal dispersion region is very well studied. The Ginzburg-Landau system [e.g., the cubic-quintic GinzburgLandau equation (CQGLE)] has been shown to support a wide variety of nonlinear phenomena in addition to stable chirped pulses, including dissipative soliton resonances [56], soliton explosions [57], pulsations [58], rains [59], soliton molecules [60], and optical bullets [61]. The demonstration of chirped pulses in the simple and passive LLE-F suggests several research directions of interest for Kerr resonators.

The numerical existence of stable chirped solitons to the LLE-F motivates the search for a corresponding closed-form 
solution. A closed-form solution allows for more efficient theoretical predictions for the behavior and existence of stable solutions. For example, for the analogous CQGLE, a particular closed-form solution for chirped pulses [53] has enabled simple design rules and predictions [32] and has been used as an ideal trial function for variational approach calculations [62]. The variational approach has been applied to the LLE-F in Ref. [34] but with a chirped Gaussian profile with strict assumptions that are violated for most realistic conditions. The results from that model have not yet been validated with full numerical simulations. Ultimately, the variational approach with the appropriate ansatz has the promise of providing an accurate and efficient approach for calculating pulsed solutions for exploring and optimizing the performance of normal dispersion Kerr resonators.

Using the variational approach for the CQGLE, researchers discovered a resonance phenomenon in which, for a particular value of parameters, the soliton energy increases indefinitely $[56,63]$. This dissipative soliton resonance is predicted to lead to remarkable performance advances for mode-locked lasers. It will be interesting and important to investigate the potential for a related dissipative soliton resonance in Kerr resonator systems.

The strongly dissipative CQGLE has also been shown to support more exotic dynamic behavior in specific parameter regimes [64]. For example, so-called exploding solitons exhibit large nonlinear instabilities in which the field can transition from a smooth localized pulse to a wildly oscillating and noisy field profile, before returning to the original smooth localized pulse. Remarkably, this exotic behavior has also been observed experimentally in mode-locked lasers $[57,65]$. The discovery of chirped pulses in the LLE-F motivates the investigation of related complex nonlinear phenomena such as exploding, creeping, and pulsating solitons in normal dispersion Kerr resonators with a filter.

In this paper, we report on the deviation of the Ikeda-type model, where the cavity is driven a one point in the resonator, from the mean-field LLE-F model, where the cavity is driven continuously around the resonator (Sec. III D). In the LLE-F system, the drive has a strong dependence on loss, suggesting that low drive powers will be achievable with high-Q cavities, as is typical with traditional anomalous dispersion Kerr resonators. In the Ikeda-type model, however, the parameters are insensitive to loss, which enables opportunities for generating frequency combs in low-Q cavities. In addition to allowing for lossy material designs, this loss insensitivity could enable large output coupling for high output energy designs.

As discussed in Sec. III D, the deviation of the system from the mean-field LLE model stems from the large intracavity evolution of chirped pulses in the resonator. This motivates further investigation into other pulse solutions featuring large intracavity evolution. For example, stretched pulse solitons are defined by periodic temporal stretching and compressing in the resonator, with breathing ratios demonstrated $>3$ [16]. The stretched-pulse soliton may therefore deviate significantly from a mean-field model, leading to unconventional parameter dependence.

Note that, while this paper is restricted to the lowest order of dispersion and self-phase modulation in a singlemode waveguide, higher-order nonlinearity and dispersion, as well as intermodal interactions, are known to stabilize pulse forms in normal dispersion Kerr resonators [23-30]. For example, in one related study, Huang et al. [34] studied the effect of low-modulation depth intrinsic spectral filtering on normal dispersion microresonators. This system features strongly frequency-dependent dissipation and dispersion including mode crossings and effective higher-order dispersion. Indeed, the authors found evidence of weakly chirped brightpulse formation experimentally and theoretically in a model including these effects. It would be interesting to determine whether highly chirped pulses could be stable in a microresonator system with or without higher-order effects as well. Regardless, mode crossings and higher-order effects may also be important for the dynamics of high energy and ultrashort chirped pulses in normal dispersion Kerr resonators with a filter and merits further investigation.

\section{CONCLUSIONS}

In this paper, we examine stable solutions to the LLE-F. We find stable highly chirped solitons over a range of parameters. The range of parameters for which stable chirped pulses exist is identified, and simple scaling laws are shown to be useful for designing practical resonators. The LLE-F model is found to deviate from the experimentally accurate Ikeda-F model for chirped pulses because of the large intracavity dynamics of these solutions. This deviation leads to a weak dependence on the system loss, contrary to conventional design rules for Kerr resonators. As described in detail in the Discussion section, the results of this study lay the foundations for investigating a variety of nonlinear dynamical phenomena, give design guidelines based on simple scaling laws for the generation of chirped pulses in Kerr resonators, and enable additional flexibility for ultrashort pulse and frequency comb generation in low-Q cavities.

\section{ACKNOWLEDGMENT}

This paper is supported by the National Institute of Biomedical Imaging and Bioengineering of the National Institutes of Health under Award No. EB028933.

\section{APPENDIX A: NUMERICAL MODEL}

The LLE-F accounts for dispersion, Kerr nonlinearity, the drive, drive frequency detuning, loss, and spectral filtering. The LLE-F is solved numerically with a standard split-step Fourier technique with the dispersive effects calculated in the Fourier domain and the nonlinear effects solved with a fourth-order Runge-Kutta method. The cavity parameters are chosen to correspond to experimentally relevant parameters [35]. For example, $\beta_{2}=9688 \mathrm{fs}^{2} / \mathrm{m}, n_{2}=3.2 \times$ $10^{-20} \mathrm{~m}^{2} / \mathrm{W}$, mode - field diameter $=8.1 \mu \mathrm{m}$, and the total loss is $20.25 \mathrm{~dB} / \mathrm{km}$ [35]. The spectral filter bandwidth is fixed, and the simulations are run for a wide range of drive power and detuning values. The waveform is numerically propagated for $52.5 \mathrm{~km}$, which is sufficient for convergence without excessive computation time. Solutions at the end of propagation with a difference between the peak and minimum intensity of $<0.1 \mathrm{~W}$ are indicated as $\mathrm{CW}$ (trivial) 


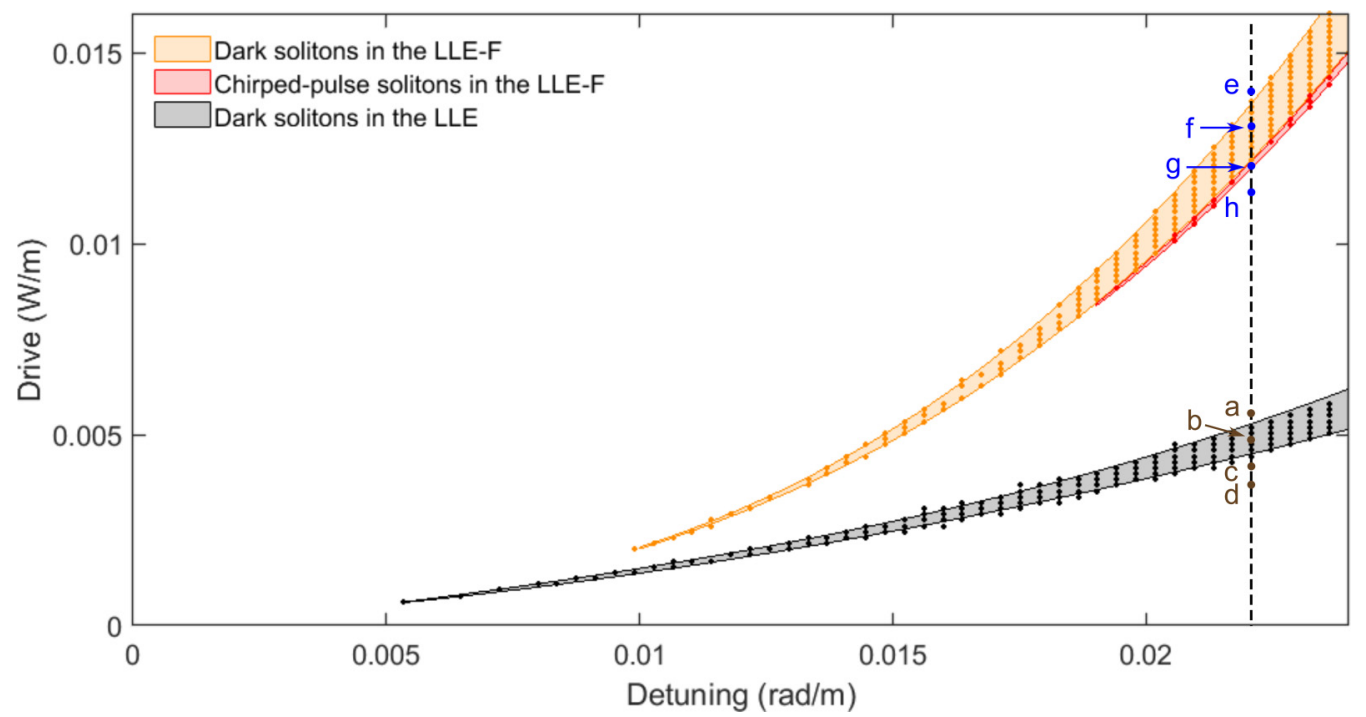

FIG. 7. Location of stable nontrivial solitons in drive-detuning parameter space with (LLE-F) and without (LLE) spectral filtering. Black points represent dark solitons of the LLE, orange points represent dark solitons of the LLE-F with a $14.5 \mathrm{~nm}$ per unit length spectral filter, and red dots represent chirped-pulse solitons in the LLE-F with the same filter. The shaded regions indicate the boundaries of each stable solution in parameter space. The dashed line and indicated points correspond to the solutions examined in Fig. 8.

by white regions in Fig. 7. Solutions with larger peak to minimum intensity differences are evaluated for convergence (as in Ref. [35]). Nontrivial converged solutions are indicated by points in Fig. 7. With no spectral filtering (LLE), the stable nontrivial solutions are dark solitons, indicated with black points in Fig. 7. With spectral filtering (LLE-F), stable nontrivial solutions include dark solitons (indicated with orange) and chirped pulses (indicted with red). The regions of existence of each solution are determined by fitting the numerically determined upper and lower boundary of the regions with a polynomial. The regions of existence between these two boundaries are shaded in color for each solution in Figs. 1 and 7. The steady-state solutions are strongly sensitive to the initial conditions. To maximize the probability of obtaining nontrivial solutions, for each choice of system parameters, several different initial conditions are evaluated, and only the nontrivial solutions are retained. Gaussian and randomly varying initial conditions can result in different solutions (e.g., see Ref. [35]), and therefore, to increase the diversity of outcomes and maximize the number of nontrivial solutions obtained in the given computation time, three Gaussian pulses with 60$\mathrm{W}$ peak power and pulse durations of 4,5 , and $6 \mathrm{ps}$; and three random intensity distributions with power varying from 0 to $120 \mathrm{~W}$ are used to obtain enough stable solutions to determine smooth boundaries defining the relevant regions of stability in parameter space (Fig. 7).

The lumped-element Ikeda-F model consists of an evolution equation describing the propagation of the slowly varying field envelope inside the resonator waveguide in addition to periodic boundary conditions. Pulse propagation in the waveguide is governed by a nonlinear Schrödinger equation as

$$
\frac{\partial A}{\partial z}=-i \overline{\frac{\beta_{2}}{2}} \frac{\partial^{2} A}{\partial t^{2}}+i \gamma|A|^{2} A,
$$

where $A$ is the slowly varying field, $z$ is the propagation coordinate, $t$ is time in the moving frame, $\overline{\beta_{2}}$ is the average group-velocity dispersion, and $\gamma$ is the nonlinearity per unit length. The interaction between the intracavity field, the drive, component loss, and the spectral filter can be described in the Fourier domain as

$$
\begin{aligned}
A_{n+1}(\omega)= & \exp \left(-i \delta_{\text {tot }}\right) A_{n}(\omega) \exp \left\{-\left[\frac{\left(\omega-\omega_{0}\right)^{2}}{f_{\text {tot }}^{2}}+\frac{\alpha_{\text {tot }}}{2}\right]\right\} \\
& +\sqrt{D_{\text {tot }}} \delta\left(\omega-\omega_{0}\right),
\end{aligned}
$$

where $\alpha_{\text {tot }}$ is the roundtrip loss, $D_{\text {tot }}$ is the total input coupled drive power, $A_{n}(\omega)$ is the spectral field at the end of the $n$th roundtrip, $A_{n+1}(\omega)$ is the spectral field at the beginning of the $(n+1)$ th roundtrip, $\omega_{0}$ is the pumping frequency, $f_{\text {tot }}$ represents the bandwidth of the spectral filter applied each roundtrip, and $\delta_{\text {tot }}$ is the total phase detuning resulting from the frequency detuned from the cavity resonance. The detuning represents a phase slip between the drive and the cavity field and can be expressed either as a boundary condition or in the propagation equation for the field. The phase detuning $\delta_{\text {tot }}$ can be expressed as a detuning per unit length $\delta$ times the cavity length $\delta_{\text {tot }}=\delta L$, giving

$$
\begin{aligned}
& A_{n+1}(\omega)=\exp (-i \delta L) A_{n}(\omega) \exp \left\{-\left[\frac{\left(\omega-\omega_{0}\right)^{2}}{f_{\text {tot }}^{2}}+\frac{\alpha_{\text {tot }}}{2}\right]\right\} \\
& +\sqrt{D_{\text {tot }}} \delta\left(\omega-\omega_{0}\right) . \\
& \text { If } A^{\prime}(z)=e^{-i \delta z} A(z) \text {, then } \\
& \frac{\partial A^{\prime}}{\partial z}=\frac{\partial A}{\partial z} e^{-i \delta z}-i \delta A^{\prime},
\end{aligned}
$$

which when combined with Eq. (A1) gives the detuned nonlinear Schrödinger equation:

$$
\frac{\partial A^{\prime}}{\partial z}=-i \frac{\overline{\beta_{2}}}{2} \frac{\partial^{2} A^{\prime}}{\partial t^{2}}+i \gamma\left|A^{\prime}\right|^{2} A^{\prime}-i \delta A^{\prime} .
$$



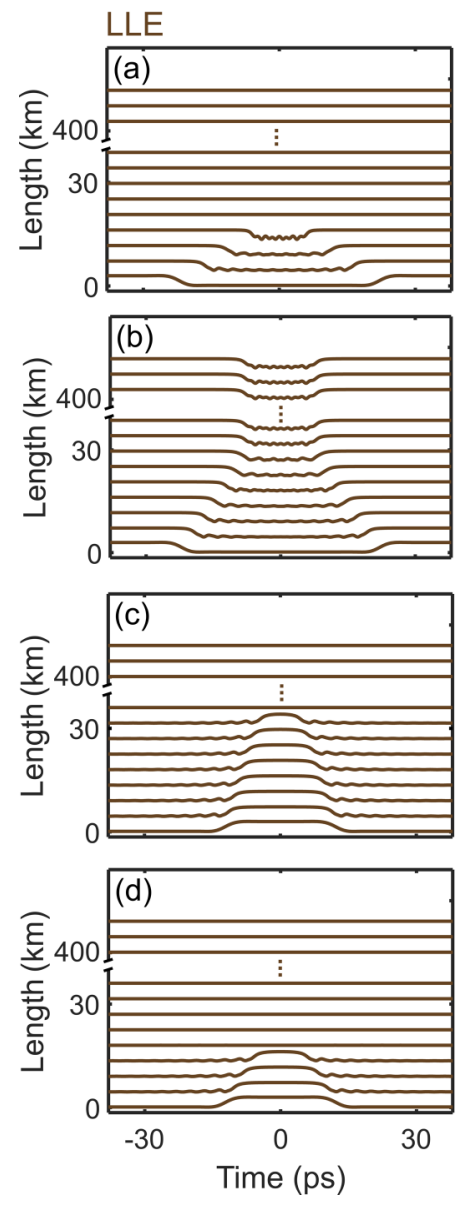
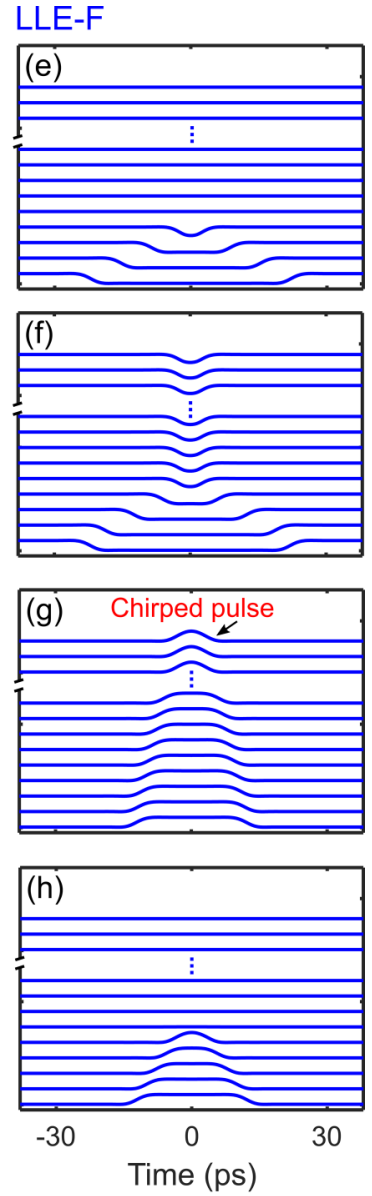

FIG. 8. Evolution to the converged solution for the LugiatoLefever equation with (LLE-F) and without (LLE) a spectral filter. The subpanels (a)-(h) correspond to the parameters with the same labels in Fig. 7. (a), (e), (d), and (h) At the highest and lowest drive powers, both systems converge to the trivial continuous wave $(\mathrm{CW})$ solution. At the drive powers from (b) and (f), both systems converge to a dark soliton solution, with a smoother field supported by the LLE-F. At lower drive powers from (b) and (f), while without a filter, only the CW solution is found, but ( $\mathrm{g}$ ) with a filter, stable chirped pulses are stabilized.

Expressing the boundary conditions for $A^{\prime}(\omega)$ yields an expression without additional phase detuning:

$$
\begin{aligned}
A_{n+1}^{\prime}(\omega)= & A_{n}^{\prime}(\omega) \exp \left\{-\left[\frac{\left(\omega-\omega_{0}\right)^{2}}{f_{\text {tot }}^{2}}+\frac{\alpha_{\text {tot }}}{2}\right]\right\} \\
& +\sqrt{D_{\text {tot }}} \delta\left(\omega-\omega_{0}\right) .
\end{aligned}
$$

The system of equations for $A^{\prime}$ can also be solved using the split-step Fourier technique. However, in this case, the drive, loss, and spectral filter are applied as a lumped element once per roundtrip. This technique was used to find the regions where stable solutions exist in Fig. 3, where the boundary fitting technique described above is also used.

\section{APPENDIX B: FORMATION OF CHIRPED PULSES}

Here, we develop some insight into the difference between dark solitons in the LLE and LLE-F as well as into the forma-
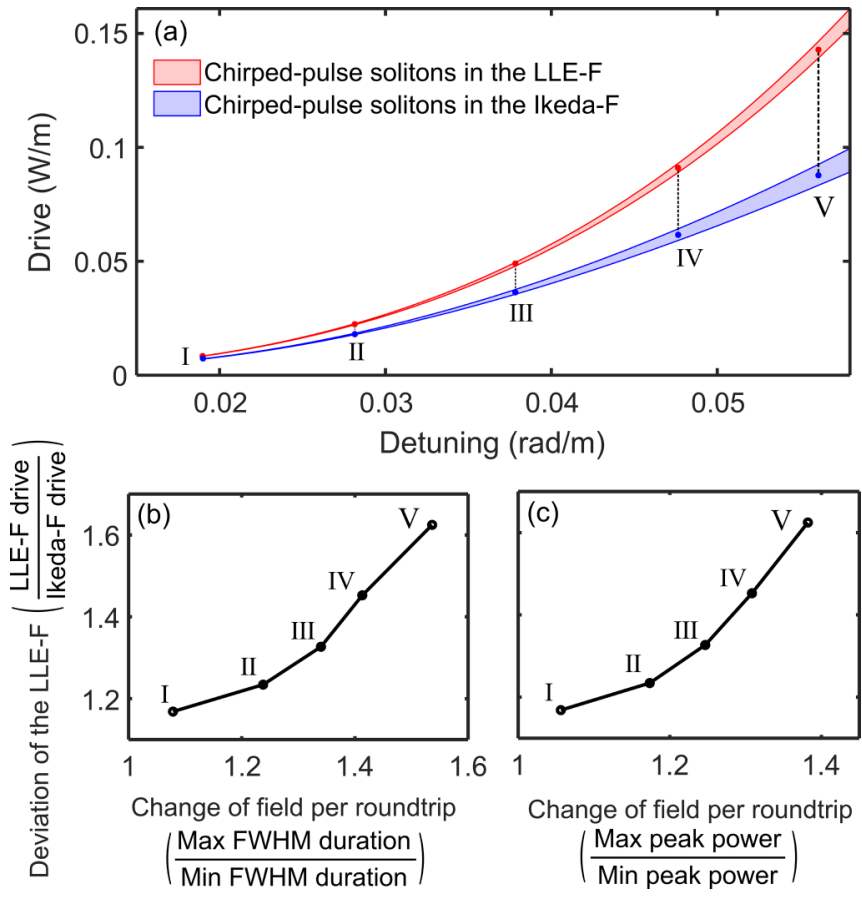

FIG. 9. Illustration of the deviation of the LLE-F from the IkedaF model. (a) Regions in the drive-detuning parameter space where chirped pulses are found in the LLE-F (red) and Ikeda-F (blue) models. Change in the required drive power between the LLE-F and Ikeda-F models as a function of (b) the ratio of the longest to the shortest pulse duration (full width at half maximum) and (c) the ratio of the highest to lowest peak power for five different detuning values with the other parameters fixed. The dashed line and indicated points in (a) correspond to the solutions examined in (b) and (c).

tion of chirped-pulse solitons unique to the LLE-F. For fixed detuning, Fig. 7 indicates that the drive power is a suitable parameter for traversing between the different solution types. We therefore examine the variation of steady-state solutions along the dashed line in Fig. 7. For a normal dispersion Kerr resonator without spectral filtering, front solutions (also known as domain walls or switching waves) often move in the reference frame of the driving field $[19,54,55,66]$. To examine the moving properties of front solutions, we initialize the simulation with a two-front intensity variation in the time domain. The equation is numerically solved with this initial condition and examined as a function of propagation distance until the waveform converges. At large drive powers without a filter [Fig. 8(a) and a from Fig. 7], the front solutions move together and vanish to converge to a CW solution. With lower drive powers [Fig. 8(b) and b from Fig. 7], the velocity of the domain walls decreases as they lock together to form a converged dark soliton. The stabilizing force has been shown previously to stem from the overlapping oscillatory tails of the domain walls $[55,67]$. With still lower drive powers [Fig. 8(c) and c from Fig. 7], the front solutions move in the opposite direction until the solution converges to a $\mathrm{CW}$ solution. The same behavior occurs at lower drive powers [Fig. 8(d) and d from Fig. 7], with a higher front velocity. Note that the intensity peak of the initial condition is either presented at the center of the window [Figs. 8(c) 
and $8(\mathrm{~d})$ ] or the edges of the window [Figs. 8(a) and 8(b)] to best emphasize the salient properties of the converged solution.

With a filter, in the LLE-F, the front behavior is altered slightly. At high drive powers [Fig. 8(e) and e from Fig. 7], as without a filter, the front solutions move together and vanish, to converge to a $\mathrm{CW}$ solution. Likewise, at slightly lower drive powers [Fig. 8(f) and f from Fig. 7], the velocity of the domain walls decreases as they lock together to form a converged dark soliton. The solutions in the LLE-F appear smoother than in the LLE without oscillatory tails.

In the LLE-F, at still lower drive powers [Fig. 8(g) and $\mathrm{g}$ from Fig. 7], the front solutions move in the opposite direction, but in this case, they lock together to converge to a bright-pulse solution. This bright-pulse solution is the chirped-pulse soliton. The spectral filter serves as a stabilizing mechanism for the two front solutions. When the drive power is even lower [Fig. 8(h) and h from Fig. 7], the domain walls move at a faster rate, and they are not stabilized into a chirped pulse but rather converge to a $\mathrm{CW}$ solution. To summarize, while the front velocity dynamics remain qualitatively the same with and without a filter, in the LLE-F, the drive power is larger, the solutions are smoother, and importantly, chirped pulses can be stabilized.

\section{APPENDIX C: VALIDITY OF THE LLE-F MODEL}

The variation of the electric field of the stable chirped pulses in the steady-state cavity is examined at five different frequency detuning values, with other parameters fixed (points in Fig. 9). For the converged solution, the ratio of the longest to the shortest pulse duration (full width at half maximum) and highest to lowest peak power is examined as a function of the deviation of the required drive from that of the Ikeda-F model [Figs. 9(b) and 9(c)]. The deviation of the models is clearly correlated to changes in the electric field with respect to pulse duration [Fig. 9(b)], peak power [Fig. 9(c)], or bandwidth (Fig. 4). The deviation of the models is larger at larger values of detuning. These results suggest that predictions based on master-equation (averaged-cavity) models should be carefully confirmed for validity in the case of large intracavity pulse evolutions.
[1] T. J. Kippenberg, A. L. Gaeta, M. Lipson, and M. L. Gorodetsky, Dissipative Kerr solitons in optical microresonators, Science 361, eaan8083 (2018).

[2] P. Marin-Palomo, J. N. Kemal, M. Karpov, A. Kordts, J. Pfeifle, M. H. P. Pfeiffer, P. Trocha, S. Wolf, V. Brasch, M. H. Anderson, R. Rosenberger, K. Vijayan, W. Freude, T. J. Kippenberg, and C. Koos, Microresonator-based solitons for massively parallel coherent optical communications, Nature (London) 546, 274 (2017).

[3] M. G. Suh and K. J. Vahala, Soliton microcomb range measurement, Science 359, 884 (2018).

[4] M. G. Suh, Q. F. Yang, K. Y. Yang, X. Yi, and K. J. Vahala, Microresonator soliton dual-comb spectroscopy, Science 354, 600 (2016).

[5] D. T. Spencer, S. H. Lee, D. Y. Oh, M.-G. Suh, K. Y. Yang, and K. Vahala, An optical-frequency synthesizer using integrated photonics, Nature (London) 557, 81 (2018).

[6] C. Bao, M. G. Suh, and K. Vahala, Microresonator soliton dualcomb imaging, Optica 6, 1110 (2019).

[7] P. Trocha, M. Karpov, D. Ganin, M. H. P. Pfeiffer, A. Kordts, S. Wolf, J. Krockenberger, P. Marin-Palomo, C. Weimann, S. Randel, W. Freude, T. J. Kippenberg, and C. Koos, Ultrafast optical ranging using microresonator soliton frequency combs, Science 359, 887 (2018).

[8] A. Dutt, C. Joshi, X. Ji, J. Cardenas, Y. Okawachi, K. Luke, A. L. Gaeta, and M. Lipson, On-chip dual-comb source for spectroscopy, Sci. Adv. 4, e1701858 (2018).

[9] F. Leo, S. Coen, P. Kockaert, S. P. Gorza, P. Emplit, and M. Haelterman, Temporal cavity solitons in one-dimensional Kerr media as bits in an all-optical buffer, Nat. Photonics 4, 471 (2010).

[10] T. Herr, V. Brasch, J. D. Jost, C. Y. Wang, N. M. Kondratiev, M. L. Gorodetsky, and T. J. Kippenberg, Temporal solitons in optical microresonators, Nat. Photonics 8, 145 (2014).
[11] N. Lilienfein, C. Hofer, M. Högner, T. Saule, M. Trubetskov, V. Pervak, E. Fill, C. Riek, A. Leitenstorfer, J. Limpert, F. Krausz, and I. Pupeza, Temporal solitons in free-space femtosecond enhancement cavities, Nat. Photonics 13, 214 (2019).

[12] P. Del'Haye, T. Herr, E. Gavartin, M. L. Gorodetsky, R. Holzwarth, and T. J. Kippenberg, Octave Spanning Tunable Frequency Comb from a Microresonator, Phys. Rev. Lett. 107, 063901 (2011).

[13] Y. K. Chembo and N. Yu, On the generation of octave-spanning optical frequency combs using monolithic whispering-gallerymode microresonators, Opt. Lett. 35, 2696 (2010).

[14] Y. Wang, M. Anderson, S. Coen, S. G. Murdoch, and M. Erkintalo, Stimulated Raman Scattering Imposes Fundamental Limits to the Duration and Bandwidth of Temporal Cavity Solitons, Phys. Rev. Lett. 120, 053902 (2018).

[15] S. M. Kelly, Characteristic sideband instability of periodically amplified average soliton, Electron. Lett. 28, 806 (1992).

[16] X. Dong, Q. Yang, C. Spiess, V. G. Bucklew, and W. H. Renninger, Stretched-Pulse Soliton Kerr Resonators, Phys. Rev. Lett. 125, 033902 (2020).

[17] Y. Li, S. Huang, B. Li, H. Liu, J. Yang, A. K. Vinod, K. Wang, M. Yu, D. Kwong, H. Wang, K. K. Wong, and C. W. Wong, Real-time transition dynamics and stability of chip- scale dispersion-managed frequency microcombs, Light Sci. Appl. 9, 52 (2020).

[18] X. Xue, Y. Xuan, Y. Liu, P. H. Wang, S. Chen, J. Wang, D. E. Leaird, M. Qi, and A. M. Weiner, Mode-locked dark pulse Kerr combs in normal-dispersion microresonators, Nat. Photonics $\mathbf{9}$, 594 (2015).

[19] B. Garbin, Y. Wang, S. G. Murdoch, G. L. Oppo, S. Coen, and M. Erkintalo, Experimental and numerical investigations of switching wave dynamics in a normally dispersive fibre ring resonator, Eur. Phys. J. D 71, 240 (2017). 
[20] C. Bao, Y. Xuan, D. E. Leaird, M. Qi, and A. M. Weiner, Dark Breathers in a Normal Dispersion Optical Microresonator, Phys. Rev. Lett. 121, 257401 (2018).

[21] F. Bessin, F. Copie, M. Conforti, A. Kudlinski, and A. Mussot, Modulation instability in the weak normal dispersion region of passive fiber ring cavities, Opt. Lett. 42, 3730 (2017).

[22] S. Coen and M. Haelterman, Modulational Instability Induced by Cavity Boundary Conditions in a Normally Dispersive Optical Fiber, Phys. Rev. Lett. 79, 4139 (1997).

[23] V. E. Lobanov, G. Lihachev, T. J. Kippenberg, and M. L. Gorodetsky, Frequency combs and platicons in optical microresonators with normal GVD, Opt. Express 23, 7713 (2015).

[24] J. K. Jang, Y. Okawachi, M. Yu, K. Luke, X. Ji, M. Lipson, and A. L. Gaeta, Dynamics of mode-coupling-induced microresonator frequency combs in normal dispersion, Opt. Express 24, 28794 (2016).

[25] Z. Li, Y. Xu, S. Coen, S. Murdoch, and M. Erkintalo, Experimental observations of bright dissipative Kerr cavity solitons and their collapsed snaking in a driven resonator with normal dispersion, Optica 7, 1195 (2020).

[26] Y. Xu, A. Sharples, J. Fatome, S. Coen, M. Erkintalo, and S. G. Murdoch, Frequency comb generation in a pulse-pumped normal dispersion Kerr mini-resonator, Opt. Lett. 46, 512 (2021).

[27] P. Parra-Rivas, S. Coulibaly, M. G. Clerc, and M. Tlidi, Influence of stimulated Raman scattering on Kerr domain walls and localized structures, Phys. Rev. A 103, 013507 (2020).

[28] P. Parra-Rivas, D. Gomila, and L. Gelens, Coexistence of stable dark- and bright-soliton Kerr combs in normal-dispersion resonators, Phys. Rev. A 95, 053863 (2017).

[29] M. G. Clerc, S. Coulibaly, and M. Tlidi, Time-delayed nonlocal response inducing traveling temporal localized structures, Phys. Rev. Res. 2, 13024 (2020).

[30] M. G. Clerc, S. Coulibaly, P. Parra-Rivas, and M. Tlidi, Nonlocal Raman response in Kerr resonators: Moving temporal localized structures and bifurcation structure, Chaos 30, 083111 (2020).

[31] A. Chong, J. R. Buckley, W. H. Renninger, and F. W. Wise, All-normal-dispersion femtosecond fiber laser, Opt. Express 14, 10095 (2006).

[32] W. H. Renninger, A. Chong, and F. W. Wise, Dissipative solitons in normal-dispersion fiber lasers, Phys. Rev. A 77, 023814 (2008).

[33] K. Kieu, W. Renninger, A. Chong, and F. W. Wise, Sub-100-fs pulses at watt-level powers from a fiber laser, Opt. Lett. 34, 593 (2009).

[34] S. W. Huang, H. Zhou, J. Yang, J. F. McMillan, A. Matsko, M. Yu, D. L. Kwong, L. Maleki, and C. W. Wong, Mode-Locked Ultrashort Pulse Generation from On-Chip Normal Dispersion Microresonators, Phys. Rev. Lett. 114, 053901 (2015).

[35] C. Spiess, Q. Yang, X. Dong, V. Bucklew, and W. Renninger, Chirped dissipative solitons in driven optical resonators, Optica 8, 861 (2021).

[36] I. H. Agha, Y. Okawachi, and A. L. Gaeta, Theoretical and experimental investigation of broadband cascaded four-wave mixing in high-Q microspheres, Opt. Express 17, 16209 (2009).

[37] N. Blow and J. Doran, Global and Local Chaos in the Pumped Nonlinear Schrödinger Equation, Phys. Rev. Lett. 52, 526 (1984).
[38] K. Ikeda, Multiple-valued stationary state and its instability of the transmitted light by a ring cavity system, Opt. Commun. 30, 257 (1979).

[39] M. Haelterman, S. Trillo, and S. Wabnitz, Dissipative modulation instability in a nonlinear dispersive ring cavity, Opt. Commun. 91, 401 (1992).

[40] L. A. Lugiato and R. Lefever, Spatial Dissipative Structures in Passive Optical Systems, Phys. Rev. Lett. 58, 2209 (1987).

[41] M. Haelterman, S. Trillo, and S. Wabnitz, Additive-modulationinstability ring laser in the normal dispersion regime of a fiber, Opt. Lett. 17, 745 (1992).

[42] Y. Wang, F. Leo, J. Fatome, M. Erkintalo, S. G. Murdoch, and S Coen, Universal mechanism for the binding of temporal cavity solitons, Optica 4, 855 (2017).

[43] S. Coen and M. Erkintalo, Universal scaling laws of Kerr frequency combs, Opt. Lett. 38, 1790 (2013).

[44] S. Coen, H. G. Randle, T. Sylvestre, and M. Erkintalo, Modeling of octave-spanning Kerr frequency combs using a generalized mean-field Lugiato-Lefever model, Opt. Lett. 38, 37 (2013)

[45] C. Godey, I. V. Balakireva, A. Coillet, and Y. K. Chembo, Stability analysis of the spatiotemporal Lugiato-Lefever model for Kerr optical frequency combs in the anomalous and normal dispersion regimes, Phys. Rev. A 89, 063814 (2014).

[46] Z. Qi, S. Wang, J. Jaramillo-Villegas, M. Qi, A. M. Weiner, G. D'Aguanno, T. F. Carruthers, and C. R. Menyuk, Dissipative cnoidal waves (Turing rolls) and the soliton limit in microring resonators, Optica 6, 1220 (2019).

[47] A. J. Scroggie, W. J. Firth, G. S. McDonald, M. Tlidi, R. Lefever, and L. A. Lugiato, Pattern formation in a passive Kerr cavity, Chaos Solitons Fractals 4, 1323 (1994).

[48] A. G. Vladimirov, M. Tlidi, and M. Taki, Dissipative soliton interaction in Kerr resonators with high-order dispersion, Phys. Rev. A 103, 063505 (2021).

[49] D. Turaev, A. G. Vladimirov, and S. Zelik, Long-Range Interaction and Synchronization of Oscillating Dissipative Solitons, Phys. Rev. Lett. 108, 263906 (2012).

[50] F. Bessin, A. M. Perego, K. Staliunas, S. K. Turitsyn, A. Kudlinski, M. Conforti, and A. Mussot, Gain-through-filtering enables tuneable frequency comb generation in passive optical resonators, Nat. Commun. 10, 4489 (2019).

[51] A. M. Perego, A. Mussot, and M. Conforti, Theory of filter-induced modulation instability in driven passive optical resonators, Phys. Rev. A 103, 013522 (2021).

[52] M. Tlidi, M. Le Berre, E. Ressayre, A. Tallet, and L. Di Menza, High-intensity localized structures in the degenerate optical parametric oscillator: Comparison between the propagation and the mean-field models, Phys. Rev. A 61, 043806 (2000).

[53] J. M. Soto-Crespo, N. N. Akhmediev, V. V. Afanasjev, and S. Wabnitz, Pulse solutions of the cubic-quintic complex Ginzburg-Landau equation in the case of normal dispersion, Phys. Rev. E 55, 4783 (1997).

[54] P. Parra-Rivas, E. Knobloch, D. Gomila, and L. Gelens, Dark solitons in the Lugiato-Lefever equation with normal dispersion, Phys. Rev. A 93, 063839 (2016).

[55] P. Parra-Rivas, D. Gomila, E. Knobloch, L. Gelens, and S. Coen, Origin and stability of dark pulse Kerr frequency combs in normal dispersion microresonators, Opt. Lett. 41, 2402 (2016). 
[56] W. Chang, A. Ankiewicz, J. M. Soto-Crespo, and N. Akhmediev, Dissipative soliton resonances, Phys. Rev. A 78, 023830 (2008).

[57] S. T. Cundiff, J. M. Soto-Crespo, and N. Akhmediev, Experimental Evidence for Soliton Explosions, Phys. Rev. Lett. 88, 073903 (2002).

[58] N. Akhmediev, J. M. Soto-Crespo, and G. Town, Pulsating solitons, chaotic solitons, period doubling, and pulse coexistence in mode-locked lasers: Complex Ginzburg-Landau equation approach, Phys. Rev. E 63, 056602 (2001).

[59] S. Chouli and P. Grelu, Soliton rains in a fiber laser: An experimental study, Phys. Rev. A 81, 063829 (2010).

[60] M. Stratmann, T. Pagel, and F. Mitschke, Experimental Observation of Temporal Soliton Molecules, Phys. Rev. Lett. 95, 143902 (2005).

[61] W. H. Renninger and F. W. Wise, Spatiotemporal soliton laser, Optica 1, 101 (2014).
[62] B. G. Bale, J. N. Kutz, A. Chong, W. H. Renninger, and F. W. Wise, Spectral filtering for high-energy mode-locking in normal dispersion fiber lasers, J. Opt. Soc. Am. B 25, 1763 (2008).

[63] N. Akhmediev, J. M. Soto-Crespo, and P. Grelu, Roadmap to ultra-short record high-energy pulses out of laser oscillators, Phys. Lett. A 372, 3124 (2008).

[64] J. M. Soto-Crespo, N. Akhmediev, and A. Ankiewicz, Pulsating, Creeping, and Erupting Solitons in Dissipative Systems, Phys. Rev. Lett. 85, 2937 (2000).

[65] S. C. V. Latas and M. F. S. Ferreira, Soliton explosion control by higher-order effects, Opt. Lett. 35, 1771 (2010).

[66] S. Coen, M. Tlidi, P. Emplit, and M. Haelterman, Convection versus Dispersion in Optical Bistability, Phys. Rev. Lett. 83, 2328 (1999).

[67] Y. Pomeau, Front motion, metastability and subcritical bifurcations in hydrodynamics, Phys. D Nonlinear Phenom. 23, 3 (1986). 